Psychological Medicine, 1977, 7, 185-187

Printed in Great Britain

\title{
EDITORIAL
}

\section{Neo-Pavlovianism and clinical psychiatry ${ }^{1}$}

Every psychiatrist is aware of the 'psychosomatic' nature of his material ; he knows that he is working with mental phenomena which he does not understand but believes to be related to the function of biological structures which can be studied by scientific methods. He is aware of a neuron and a brain packed with cells which are the sine qua non for mental phenomena - but he is largely ignorant of the mechanisms. In two generations he has seen how changes in this structure from toxic or other causes from without can induce mental disease, e.g. beri-beri, pellagra, dementia paralytica, or how nervous function is dependent upon causes from endocrines within, e.g. in hypothyroidism, acromegaly, etc.

Beyond these demonstrable material changes, there lies an enormous field in which mental life is related to symbols. In order to study this area the psychiatrist does not have to solve the age-old question which has baffled philosophers for 3000 years, viz. the transition of the subjective from the objective. We have hardly any better answer today than Aristotle had 2500 years ago. However, Pavlov (1928) took an enormous stride forward when he devised a quantitative measure for 'psychical' phenomena which he referred to as dependent upon two states, excitation and inhibition. The study was thus put on a scientific, though limited, basis at the expense of rejecting the subjective. In separating the subjective and the objective it was possible to see each with more clarity; while still recognizing that there is a subjective element in all human communication, the separation of the subjective from the objective provides a quantitative measure that in human terms is more universal, less individual and therefore more useful for science.

The key to this type of study is the statement of William James and, before him, Thomas Huxley : 'No psychosis without neurosis', i.e. for every mental event there is a corresponding neural one. I shall not here introduce some of my doubts as to the ultimate truth of this statement. Though it may not be completely true, its application is as appropriate to psychophysiology as are the Newtonian laws to physics. Without denying the value of anatomical and biochemical data, I shall outline here principles applicable to psychiatry obtained from the objective study of symbolic phenomena by means of the conditional reflex methodology.

After working for two decades with the conditional reflexes, Pavlov (1928, p. 339) observed in his dogs a neurotic state consequent to imposing upon the dog an insoluble problem of differentiation (discrimination) between similar signals, one excitatory, the other inhibitory. It was his opinion that the difficulty of differentiation produced the neurosis. Later he saw from further experiments that neurotic development was related to the individual, to type. Another significant finding of Pavlov had to do with the disturbance of the relations of the intensity of the conditional reflexes, the paradoxical phases, which have been elaborated by Sargant (1957) to give a theoretical basis for much psychopathology.

Studying experimental neurosis in several dogs over their lifespan - a decade, 14, or 18 years - I have found that although difficult differentiation may upset a hungry dog for some days or longer, it is not as potent a cause as other factors. As already mentioned, Pavlov saw from his experience the importance of the individual dog and in my work this has also seemed paramount, outweighing the effect of difficult differentiation. In my study of the experimental neurosis (1944), there appeared several new phenomena not mentioned by Pavlov. First, though the conflict began in the food centre, it gradually spread to involve other functions - gastrointestinal, cardiovascular, genitourinary, especially the sexual. Freud thought that in the human being conflicts arise chiefly in the sexual sphere; but my studies in the dog show that the disturbance spreads from another focus to

1 Address for correspondence : Dr W. Horsley Gantt, Veterans Administration Hospital, Perry Point, Maryland 21902 , USA. 
involve the sexual system in a nonspecific way: the conflict in the food centres spreads to many other physiological systems.

It was also apparent in my studies that the influence of the experimenter, the 'Effect of Person', is much more important in inducing neurotic disturbances than is difficult differentiation. In some neurotic dogs the presence of the Person has the same effects as the signals used in the difficult differentiation; the Person brings out all the anxiety, the tachycardia, the asthmatic-like breathing, the pollakiuria, the pathological sexual erections. On the other hand, the Person may have a paradoxical influence; for when he pets the dog, the symptoms that he has produced are eliminated. Using the heart rate as a measure we are enabled to see the 'Effect of Person', although this is not observable in the dog's general behaviour. Though the dog stands quietly his heart rate may be accelerated $100 \%$ simply by the presence of the experimenter (Gantt et al. 1966).

Not only did we see the 'Effect of Person' in the neurotic dogs, but in all dogs there is an 'Effect of Person'. In psychiatry we recognize the influence of one individual on another, but we find it throughout the animal kingdom; the 'Effect of Person' is almost universal. However, in other neurotic dogs, a highly neurotic strain bred and studied by O. D. Murphree (Murphree \& Newton, 1971), these dogs paradoxically have a greatly diminished 'Effect of Person' compared with the normal.

Beginning in 1939 we added the heart rate as a measure of the conditional reflex, recording it along with respiration, secretion and movement (Gantt, 1960). The cardiac component, as well as the respiratory, gave us a view of what goes on inside the organism, often hidden from ordinary, casual observation. The cardiovascular components of heart rate, blood pressure, and blood flow reveal a picture frequently opposite to the motor behaviour. The cardiac conditional reflex as a rule forms more quickly than do the secretory and the motor components, but once formed it is very difficult to eradicate. For this difference I use the term schizokinesis (Gantt, 1953a, 1953 b, 1974). It represents a split in the functions of the organism. Although appearing quiet to casual observation, the individual may be violently agitated within, as revealed in the cardiovascular system. Indeed, schizokinesis may be an index to the susceptibility of an individual to nervous breakdown under stress and thus constitute a basis for prediction and a preventive psychiatry. Khananashvili, the successor to Pavlov in the Institute of Experimental Medicine in Leningrad, has found schizokinesis a reliable prognostic sign of incipient nervous disturbance (M. M. Khananashvili, 1975, personal communication).

In the long-term study of dogs that were worked with for one or more years and then rested we saw that in the interim there was often a development of symptoms, a spread to new systems that had not been affected in the earlier sessions, symptoms which could be demonstrated as related to the old focus, but which were formed in the resting period. As this phenomenon seemed to occur entirely from forces within the organism, independently of the present external environment, we gave it the name of autokinesis. In the neurotic dog we saw clearly what is well-known to occur in life : one experience or episode may change the whole future through continual development from the implanted excitation. In the germination of a seed there is development from energy within. As a phenomenon in pathology and as a change in the nervous elements and their connections, little or no work has been done with this concept. Here a whole new area awaits a scientific method for investigation.

The tendency in the field of behaviour has been to show that through both operant and so-called Pavlovian (classical) conditioning, every function is capable of becoming a conditional reflex. Exploring viscera other than those necessary for immediate action (e.g. gastrointestinal, cardiovascular, motor) we find that those functions connected with the steady state essential for homeostasis cannot be conditioned. To define modifiable behaviour, the limitations should be known, viz. the respective roles of modifiable behaviour and of relatively unmodifiable genetic functions. The relation of an organ to its function in behaviour and body economy is what I have called Organ-system responsibility (Gantt, 1972).

These are some of the fundamental principles in psychophysiology and psychopathology that have appeared in the course of the development of my science first learned in the laboratory of Ivan 
Petrovich Pavlov. They help us to understand the patient and the development of his pathology, and by this understanding we may be led to a more successful therapy. Person must be ranked along with food, shock, and sex as a physiological (as well as psychological) stimulus. From a half century of laboratory work we see therefore emerging, in addition to Pavlov's contributions, in the most complex biological matrix, a few simple laws - schizokinesis, autokinesis, centrokinesis, 'Effect of Person', organ-system responsibility - all of which have precise physiological measures, and are applicable to clinical psychiatry.

W. HORSLEY GAN'TT

\section{REFERENCES}

Gantt, W. H. (1944). Experimental Basis for Neurotic Behavior. Hoeber \& Co.; New York.

Gantt, W. H. (1953a). Principles of nervous breakdown schizokinesis and autokinesis. Annals of the New York Academy of Sciences 56, 143-163.

Gantt, W. H. $(1953 b)$. The physiological basis of psychiatry : The conditional reflex. In Basic Problems in Psychiatry (ed. J. Wortis), pp. 52-89. Grune \& Stratton; New York.

Gantt, W. H. (1960). Cardiovascular component of the conditional reflexes to pain, food and other stimuli. Physiological Reviews Supplement 440 (II), 266-291.

Gantt, W. H. (1972). Organ-system responsibility, homeostasis and the conditional refiex. Conditional Reflex $7(1)$, $1-10$
Gantt, W. H. (1974). Autokinesis, schizokinesis, centrokinesis and organ-system responsibility: Concepts and definition. Pavlovian Journal of Biological Science 9(4), 187-191.

Gantt, W. H., Newton, J. E. O., Royer, F. L. \& Stephens, J. (1966). The effect of person. Conditional Reflex 1, 18-35.

Murphree, O. D. \& Newton, J. E. O. (1971). Cross breeding a special handling on genetically nervous dogs. Conditional Reflex 6(3), 129-136.

Pavlov, I. P. (1928). Lectures on Conditional Reflexes (trans. and ed. W. H. Gantt). International Publishers : New York. Sargant, W. (1957). Battle for the Mind. Doubleday: New York. 